\title{
Human cytomegalovirus induces apoptosis in neural stem/progenitor cells derived from induced pluripotent stem cells by generating mitochondrial dysfunction and endoplasmic reticulum stress
}

Hiroyuki Nakamura ${ }^{1 *}$, Huanan Liao ${ }^{1}$, Kahori Minami ${ }^{2}$, Masashi Toyoda $^{2}$, Hidenori Akutsu ${ }^{2}$, Yoshitaka Miyagawa $^{3}$, Hajime Okita ${ }^{3}$, Nobutaka Kiyokawa ${ }^{3}$, Akihiro Umezawa ${ }^{2}$, Ken-Ichi Imadome ${ }^{1}$, Naoki Inoue ${ }^{4}$ and Shigeyoshi Fujiwara ${ }^{1}$

\begin{abstract}
Background: Congenital human cytomegalovirus (HCMV) infection, a leading cause of birth defects, is most often manifested as neurological disorders. The pathogenesis of HCMV-induced neurological disorders is, however, largely unresolved, primarily because of limited availability of model systems to analyze the effects of HCMV infection on neural cells.

Methods: An induced pluripotent stem cell (iPSC) line was established from the human fibroblast line MRC5 by introducing the Yamanaka's four factors and then induced to differentiate into neural stem/progenitor cells (NSPCs) by dual inhibition of the SMAD signaling pathway using Noggin and SB-431542.

Results: iPSC-derived NSPCS (NSPC/iPSCs) were susceptible to HCMV infection and allowed the expression of both early and late viral gene products. HCMV-infected NSPC/iPSCs underwent apoptosis with the activation of caspase-3 and -9 as well as positive staining by the terminal deoxynucleotidyl transferase-mediated dUTP nick-end labeling (TUNEL). Cytochrome $\mathrm{c}$ release from mitochondria to cytosol was observed in these cells, indicating the involvement of mitochondrial dysfunction in their apoptosis. In addition, phosphorylation of proteins involved in the unfolded protein response (UPR), such as PKR-like eukaryotic initiation factor 2a kinase (PERK), c-Jun NH2terminal kinase (JNK), inositol-requiring enzyme 1 (IRE1), and the alpha subunit of eukaryotic initiation factor 2 (elF2a) was observed in HCMV-infected NSPC/iPSCs. These results, coupled with the finding of increased expression of mRNA encoding the C/EBP-homologous protein (CHOP) and the detection of a spliced form of X-box binding protein 1 (XBP1) mRNA, suggest that endoplasmic reticulum (ER) stress is also involved in HCMV-induced apoptosis of these cells.
\end{abstract}

Conclusions: iPSC-derived NSPCS are thought to be a useful model to study HCMV neuropathogenesis and to analyze the mechanisms of HCMV-induced apoptosis in neural cells.

Keywords: Human cytomegalovirus, iPS cells, Neural stem/progenitor cells, Apoptosis, ER stress

\footnotetext{
*Correspondence: nakamura-hry@ncchd.go.jp

1 Department of Infectious Diseases, National Research Institute for Child

Health and Development, 2-10-1 Okura, Setagaya-ku, Tokyo 157-8535, Japan

Full list of author information is available at the end of the article
} 


\section{Background}

Congenital cytomegalovirus (CMV) infection is a major cause of birth defects resulting mainly from primary CMV infection during pregnancy. At birth, approximately 5 to $10 \%$ of congenitally infected newborns are estimated to be symptomatic exhibiting multi-organ disorders including neurological defects such as mental retardation, sensorineural hearing loss, and microencephaly [1,2]. A latest study showed that if laboratory findings including those from magnetic resonance imaging (MRI) images of the brain are considered, up to $30 \%$ of congenitally infected newborns exhibit some abnormal signs [3]. Sixty to $90 \%$ of congenitally infected children who are symptomatic at birth, and 10 to $15 \%$ of those who are asymptomatic at birth develop one or more long-term sequelae. Although CMV infects a wide variety of cell types, infection of the nervous system gives most serious and long-lasting damages to the host.

As a part of understanding the HCMV neuropathogenesis, it is important to scrutinize the cellular response to CMV infection in neural cells. Some human neural cell lines can be infected with HCMV with different permissiveness to HCMV gene expression and replication [4-7]. A recent study has shown that neural progenitor cells isolated from developing human brain tissues are susceptible to CMV infection and undergo apoptosis following infection $[8,9]$. However, the amount of neural cells obtainable from human brain tissues is limited.

Pluripotent stem cells, including embryonic stem cells (ESCs) and induced pluripotent stem cells (iPSCs), are characterized by the ability to differentiate into tissues derived from any of the three embryonic germ layers. Recent advances in the method to induce efficient differentiation of either ESCs or iPSCs into specific cell lineages offer an opportunity to establish model systems for viral infections of various cell types, including neural cells. Furthermore, differentiated cells derived from pluripotent stem cells are obtainable in potentially unlimited amounts. Previous works revealed that while mouse ESCs are not susceptible to murine CMV (MCMV), NSPCs that are differentiated from them are susceptible and their proliferation and differentiation are suppressed by MCMV [10-13]. Experiments with human ESCs are, however, complicated with ethical problems.

In this study, to analyze the pathological effects of HCMV on neural cells, we prepared NSPCs from human iPSCs and examined whether NSPCs are susceptible to HCMV infection. The results indicated that NSPCs are susceptible to HCMV infection and undergo apoptosis caused by mitochondrial dysfunction and endoplasmic reticulum (ER) stress.

\section{Methods}

\section{Cells and viruses}

The human fetal lung fibroblast MRC5 was grown in Dulbecco's modified Eagle's medium (DMEM) supplemented with $10 \%$ fetal bovine serum (FBS; Invitrogen, Carlsbad, CA). The human foreskin fibroblast cell line hTERT-BJ1 immortalized with the human telomerase reverse transcriptase (Clontech, Palo Alto, CA) was grown in a medium consisting of 4 parts of DMEM and 1 part of medium 199 (Sigma) supplemented with 10\% FBS, $1 \mathrm{mM}$ sodium pyruvate (Sigma), and $2 \mathrm{mM}$ glutamine (Invitrogen). HCMV laboratory strain Towne (ATCC VR977) was propagated in hTERT-BJ1 cells. The human iPSC line MRC-iPS-25 that was established from MRC5 by retroviral vector-mediated transduction of the c-Myc, Oct4, Klf4, and Sox2 genes [14,15] were cultured on mitomycin C-treated mouse embryonic fibroblasts (MEFs) in an iPSC medium consisting of Knockout DMEM/F12 (Invitrogen) supplemented with non-essential amino acids $(0.1 \mathrm{mM}$, Invitrogen), glutamax I (1 mM, Invitrogen), 20\% Knockout Serum Replacement (Invitrogen), $\beta$-mercaptoethanol (55 $\mu \mathrm{M}$, Invitrogen) and basic fibroblast growth factor (10 ng/mL; Peprotech, Rocky Hill, NJ).

\section{Induced differentiation on iPSCs into neural stem cells}

MRC-iPSC-25 cells cultured under feeder-free conditions were induced to differentiate into neural stem/progenitor cells (NSPCs) by the method of dual inhibition of the SMAD signaling pathway described previously [16]. In brief, feeder-free iPSCs were treated with the mTeSR1 medium (StemCell Technologies, Vancouver, BC, Canada) containing Y27632 (Wako Pure Chemicals, Osaka, Japan) and maintained with a daily medium change for 4 days. Then the medium was replaced with iPSC medium supplemented with SB431542 (10 nM, Wako Pure Chemicals) and Noggin $(500 \mathrm{ng} / \mathrm{ml}$, Wako Pure Chemicals). This date was designated day 0 . On day 2 , culture medium was replaced with a medium consisting of 3 parts of iPSC medium and 1 part of N2 medium (Knockout DMEM/F12 containing 1× N2 supplement) supplemented with SB431542 (10 nM) and Noggin $(500 \mathrm{ng} / \mathrm{ml})$. On day 4 , culture medium was replaced with a medium consisting of 1 part of iPSC medium and 1 part of N2 medium supplemented with SB431542 $(10 \mathrm{nM})$ and Noggin $(500 \mathrm{ng} / \mathrm{ml})$. On day 6, cells were expanded in StemPro NSC SFM (Invitrogen). MRC-iPSC-25 cells cultured under feeder-free conditions and NSPC/ iPSCs were infected with the Towne strain HCMV at a multiplicity of infection (MOI) of 1 plaque forming unit (PFU) per cell. To detect infectious virions produced from HCMV-infected NSPC/iPSCs, supernatant was collected and replaced with fresh medium every two days after infection. hTERT-BJ1 cells were inoculated with the supernatant and examined by IFA for expression of IE1/IE2.

\section{Antibodies}

Antibodies used were as follows: rabbit anti-Sox2, rabbit anti-Nanog, rabbit anti-Oct-4, rabbit anti-cleaved caspase- 
3, rabbit anti-cleaved caspase-9, rabbit anti-phosphoeIF2 $\alpha$ (Ser51), rabbit anti-phospho-PERK (Thr980), and rabbit anti-phospho-SAPK/JNK (Thr183/Tyr185)(Cell Signaling Technology, Beverly, MA); mouse anti-CMV IE1/ IE2, rabbit anti-Musashi-1, and rabbit anti-cytochrome c (Millipore, Temecula, CA); rabbit anti-Nestin and mouse anti- $\alpha$-tubulin (Sigma-Aldrich, St. Louis, MO); rabbit antiPax6 (Covance, Princeton, NJ), mouse anti-CMV gB (Abcam, Cambridge, MA); mouse anti-pp65 (Virusys Corporation, Sykesville, MD); rabbit anti-phosphorylated IRE1 $\alpha$ (Pierce/Thermo Scientific, Rockford, IL); Alexa Fluor 488-conjugated goat anti-mouse IgG and Alexa Fluor 594-conjugated goat anti-rabbit IgG (Molecular Probes, Eugene, OR); horseradish peroxidase-conjugated donkey anti-rabbit IgG and horseradish peroxidaseconjugated sheep anti-mouse IgG (GE Healthcare, UK).

Immunofluorescence microscopy and immunoblot analysis Cells were fixed with $4 \%$ parafolmaldehyde in PBS (Wako chemicals) at room temperature (RT) for $15 \mathrm{~min}$. After fixation, cells were treated with $1.0 \%$ Triton X-100 in PBS for $15 \mathrm{~min}$ at RT and blocked with $10 \%$ goat serum in PBS for $30 \mathrm{~min}$. Cells were incubated with the primary antibody at $4^{\circ} \mathrm{C}$ overnight, followed by washing in PBS and incubation with the corresponding secondary antibody at $37^{\circ} \mathrm{C}$ for $1 \mathrm{~h}$. Nuclei were stained with DAPI. For the assessment of cell death, terminal deoxynucleotidyl transferase (TdT)-mediated dUTP nick-end labeling (TUNEL) assay was performed according to the manufacturer's instructions (Roche). Immunoblot analyses were performed as described previously [17].

\section{Reverse transcriptase (RT)-PCR and real-time quantitative RT-PCR}

Total RNA was isolated from mock- or HCMV-infected cells using TRIzol reagent (Invitrogen). Reverse transcription was performed on each RNA sample $(5 \mu \mathrm{g})$ using SuperScript III First-Strand Synthesis System for RT-PCR (Invitrogen). Primer sequences are shown in Table 1. RT-PCR products were resolved by electrophoresis on $2 \%$ agarose gel and then visualized by ultraviolet illumination after ethidium bromide staining. Real-time quantitative RT-PCR was performed using TaqMan ${ }^{\mathrm{TM}}$ Universal Master Mix II with UNG (Applied Biosystems) on a StepOne Plus PCR System (Applied Biosystems). Amplifications were achieved in a final volume of $25 \mu \mathrm{l}$ containing TaqMan probes labeled with FAM on the 5'end and MGB on the 3'-end. The primers and probes for UL136 were: forward primer, 5'-GGCCGTTGAAC GGAGCTAT-3' and reverse primer, 5'-CCATTTCCAC CGTGTCGAA-3', and TaqMan probe, 5'-FAM-TACT ACGGCAGCGGCT-MGB-3'. The forward and reverse primers and reporter probes for HCMV IE1, UL89, and Human G6PD were described previously [18].
Table 1 List of primer sequences for RT-PCR

\begin{tabular}{|c|c|c|}
\hline Gene & Forward primer & Reverse primer \\
\hline $\mathrm{EE} 1^{*}$ & ATGGAGTCCTCTGCCAAGAG & ATTCTATGCCGCACCATGTCC \\
\hline $\mathrm{IE} 2^{*}$ & ATGGAGTCCTCTGCCAAGAG & CTGAGACTTGTTCCTCAGGTCCTG \\
\hline VIL-10* & ATGCTGTCGGTGATGGTCTCTTCC & CTTTCTCGAGTGCAGATACTCTTCG \\
\hline UL36* & GACCTACGGGACACGCTGATG & TGTGGAAGTGGTCGCAGTGAC \\
\hline UL38 & GACTACGACCACGCATAGCA & GGGAACAGAGCGTTCCAATA \\
\hline pp65 & CGCAACCTGGTGCCCATGG & CGTITGGGTTGCGCAGCGGG \\
\hline$a^{a} \circ g^{*}$ & GCTTGCCTTGCTTTGAAGCA & TTCTTGACCGGGACCTTGTC \\
\hline Oct-4 & GAGCAAAACCCGGAGGAGT & TTCTCTITCGGGCCTGCAC \\
\hline Sox1 & GCGGAAAGCGTTTCTTTG & TAATCTGACTTCTCCTCCC \\
\hline Sox2 & ATGCACCGCTACGACGTGA & CTITTGCACCCCTCCCATTT \\
\hline Pax6* & AACAGACACAGCCCTCACAAACA & CGGGAACTTGAACTGGAACTGAC \\
\hline Nestin* & CAGCGTTGGAACAGAGGTTGG & TGGCACAGGTGTCTCAAGGGTAG \\
\hline $\mathrm{MAP}^{*}$ & CCACCTGAGATTAAGGATCA & GGCTTACTITGCTTCTCTGA \\
\hline$=A P^{*}$ & GTACCAGGACCTGCTCAAT & CAACTATCCTGCTTCTGCTC \\
\hline OSP* & ACTGCTGCTGACTGTTCTTC & GTAGAAACGGTITCACCAA \\
\hline $\mathrm{XBP} 1^{*}$ & CCTTGTAGTTGAGAACCAGG & GGGGCTTGGTATATATGTGG \\
\hline $\mathrm{CHOP}^{*}$ & TGGAAGCCTGGTATGAGGAC & TCACCATTCGGTCAATCAGA \\
\hline$\beta$-actin* & ACCATGGATGATGATATCGC & TCATTGTAGAAGGTGTGGTG \\
\hline $\mathrm{GAPDH}^{*}$ & CCACCCATGGCAAATTCCATGGCA & TCTAGACGGCAGGTCAGGTCCACC \\
\hline
\end{tabular}

Asterisks $\left({ }^{*}\right)$ indicate that amplified fragments contain splicing junctions. Amplified fragments for UL38, pp65, Oct-4, Sox1, and Sox2 did not contain splicing junctions, and therefore control experiments without reverse transcriptase confirmed the RNA origin of the PCR products.

\section{Results}

Preparation of human iPSC-derived neural stem/progenitor cells

Figure 1A demonstrates that MRC-iPS-25 cells have a typical iPSC colony morphology. The expression of pluripotency markers of iPSCs such as Nanog and Oct-4 in MRC-iPS-25 cells was confirmed by indirect immunofluorescence assay (IFA) (Figure 1B). The HCMV-encoded proteins IE1/IE2 were not detected in MRC-iPS-25 cells following inoculation with the virus, indicating that MRC-iPS-25 cells are either not susceptible to HCMV infection or do not support expression of the IE genes (Figure 1B).

$\mathrm{NSPC} / \mathrm{iPSC}$ prepared by induced differentiation of MRC-iPS-25 cells proliferated in a monolayer and displayed a rounded, immature neural morphology (Figure 1A). IFA (Figure 1C) showed that NSPC/iPSCs expressed the NSC markers Nestin, Sox2, and Pax6, indicating that NSPC/ iPSCs have the authentic NSPC phenotype.

\section{In vitro HCMV infection of iPSC-derived NSPCs}

To examine the susceptibility of NSPC/iPSCs to HCMV infection, these cells were inoculated in vitro with the HCMV Towne strain at an MOI of 1 PFU per cell (Figure 2A). On the second day post-infection (dpi), NSPC/iPSCs started to show morphological changes including increased cell volume and cell fusion, and the number of cells with these 

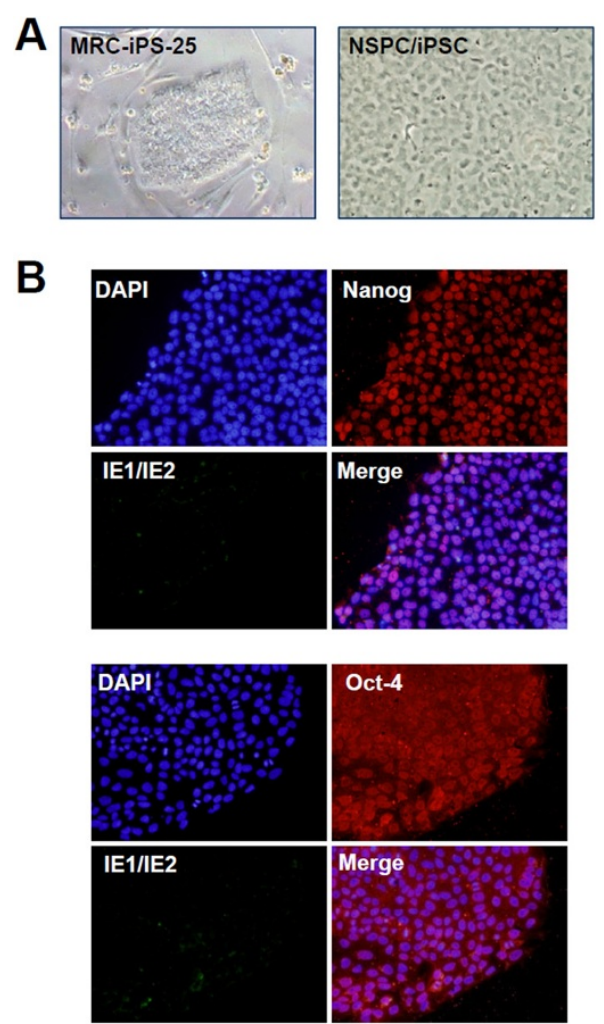
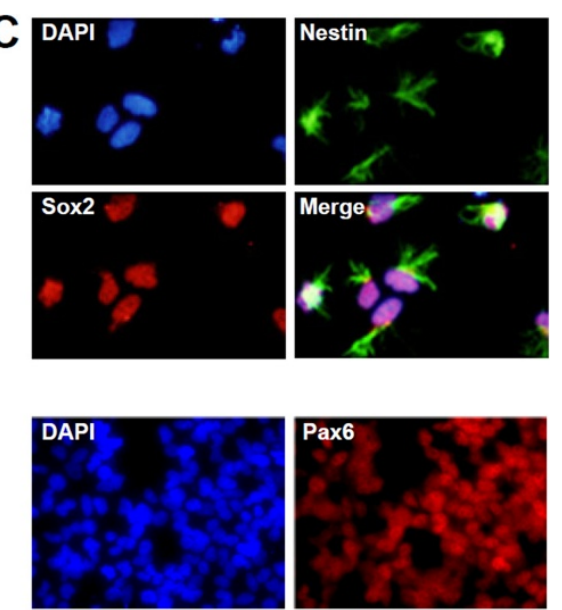

Figure 1 Differentiation of MRC-iPS-25 cells to neural stem/progenitor cells. (A) Phase-contrast images of MRC-iPS-25 cells cultured on a feeder layer of MEFs (left) and NSPC/iPSCs (right). (B) Immunofluorescence analysis of Towne-infected MRC-iPS-25 cells cultured under feeder-free conditions at 2 dpi stained with primary antibodies for pluripotent markers (Nanog or Oct-4) and HCMV IE1/IE2 proteins. Antigen proteins were detected with Alexa Fluor 488-conjugated goat anti-mouse IgG or Alexa Fluor 594-conjugated goat anti-rabbit IgG antibody. Nuclei were stained with DAPI. (C) Immunofluorescence analysis of NSPC markers Nestin, Sox2, and Pax6 in NSPC/iPSCs. NSPC/iPSCs were fixed and reacted with antiNestin (green), anti-Sox2 (red), and anti-Pax6 (red) antibodies, followed by detection with secondary antibodies. Immunofluorescence signals were obtained using a fluorescence microscope IX71. Representative results from three independent experiments are shown.

changes increased until 7 dpi (Figure 2A). To examine whether NSPC/iPSCs were capable of supporting HCMV gene expression, total RNA extracted from the infected NSPC/iPSCs was analyzed by RT-PCR. As shown in Figure 2B, mRNAs encoding IE1, IE2, vIL-10, and pp65 as well as those encoding HCMV anti-apoptotic proteins, such as UL36 and UL38, were detected. The kinetics of HCMV gene expression was analyzed by quantitative real-time RTPCR (Figure 2C). IE1 mRNA was detected first on $1 \mathrm{dpi}$ and increased steadily until 5 dpi. mRNAs for UL89 and UL136 were detected somewhat later and increased gradually until $7 \mathrm{dpi}$. The results showed the NSPC/iPSCs are susceptible to HCMV infection and allow the expression of several viral genes of both early and late functions.

Expression of HCMV genes in NSPC/iPSCs was evaluated at the protein level by immunoblot analysis on day $1,2,5$, and 7 following HCMV infection. As shown in Figure 2D, the immediate-early protein IE1 was first detected at $1 \mathrm{dpi}$ and its level increased until $5 \mathrm{dpi}$. Another immediate-early protein IE2 was detected a little later, becoming visible at 5 dpi. The expression of the HCMV lower matrix protein pp65 (ppUL83), already visible at $1 \mathrm{dpi}$, was markedly elevated at 5 and $7 \mathrm{dpi}$. The HCMV envelope glycoprotein B (gB; UL55) was detected at 5 to $7 \mathrm{dpi}$. Thus the expression of HCMV proteins of both immediate-early and late functions was demonstrated in NSPC/iPSCs.

We next examined the expression of cellular mRNAs encoding the pluripotency and neural differentiation markers (Figure 2E). Expression of the iPSC markers Nanog and Oct-4 remained at low levels following infection with HCMV, although that of Nanog tapered. While expression of the NSPC markers Sox2 and Pax6 were kept at high levels following HCMV infection, that of another NSPC marker Nestin was markedly suppressed at $7 \mathrm{dpi}$. In addition, expression of the neuronal marker microtubule-associated protein 2 (MAP2), the astrocyte marker glial fibrillary acidic protein (GFAP), and the oligodendrocyte marker oligodendrocyte-specific protein (OSP) was detected at low levels. Interestingly, Sox1, a marker specific to the neuroectodermal lineages [19], was markedly upregulated following infection with HCMV. Expression of the NSPC markers was evaluated 

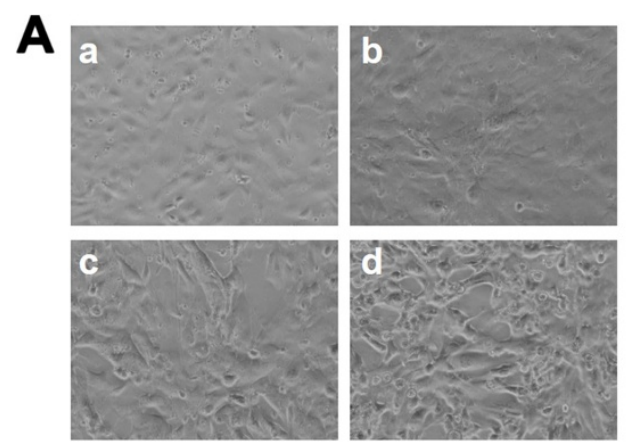

C.

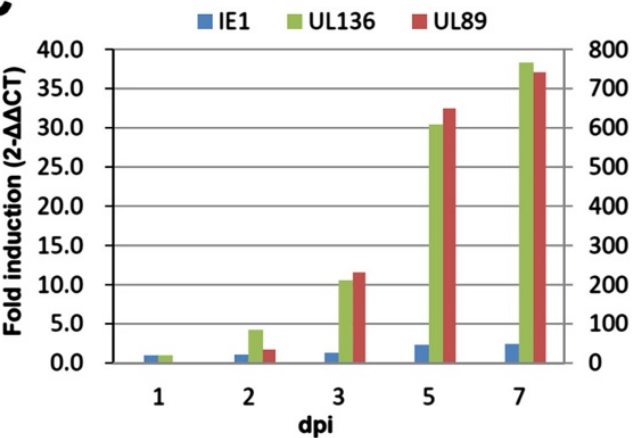

E

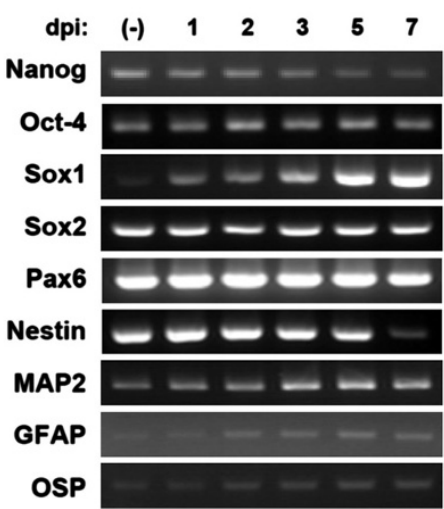

B

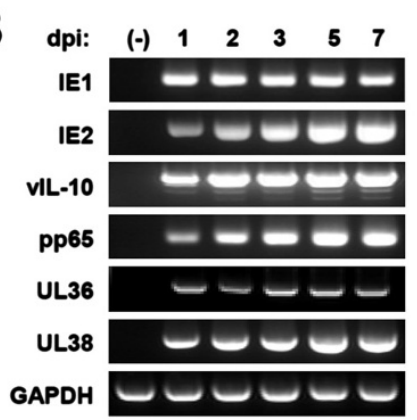

D

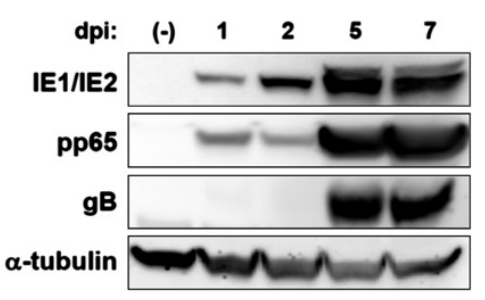

$\mathbf{F}$

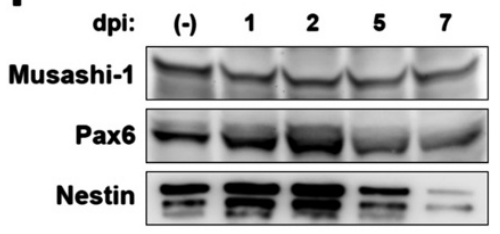

G
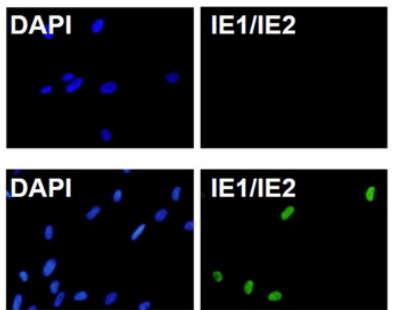

Figure 2 Analysis on the expression of viral and cellular gene products in NSPC/iPSCs. (A) Morphological changes of Towne-infected NSPC/iPSCs were observed under the inverted microscope before infection (a), 2 dpi (b), 5 dpi (c), and 7 dpi (d). (B) RT-PCR analysis of HCMVencoding gene expression. Total RNAs isolated from NSPC/iPSCs harvested before (-) HCMV infection or at 1, 2, 3, 5, and 7 dpi with HCMV Towne strain were subjected to RT-PCR assays. GAPDH gene expression was assayed for the control. (C) The kinetics of mRNA expression for IE1, UL89, and UL136 in Towne-infected NSPC/PSCs was examined by real-time quantitative RT-PCR assay. The mRNA expression was normalized to that of G6PDH gene. Real-time PCR data was analyzed by the 2- $\triangle \triangle C T$ method. The fold induction was calculated as the ratio of mRNA levels detected at each time point to that detected at $1 \mathrm{dpi}$. The $y$-axis represents fold induction of IE1 and UL136 mRNA (left y-axis) and UL89 mRNA (right y-axis). (D) Immunoblot analysis of HCMV protein expression in HCMV-infected NSPC/iPSCs. Whole-cell lysates of NSPC/iPSCs harvested before (-) HCMV infection or at 1, 2, 5, and 7 dpi with HCMV Towne strain were separated by SDS-PAGE and analyzed by immunoblotting with antibodies against IE1/IE2, pp65, gB, and a-tubulin. (E) RT-PCR analysis of pluripotency and neural differentiation marker gene expression in HCMV-infected NSPC/iPSCS. (F) Immunoblot analysis of neural differentiation marker protein expression in HCMV-infected NSPC/iPSCs. Whole-cell lysates of NSPC/PSCS were analyzed by immunoblotting with antibodies against Musashi-1, Pax6, and Nestin. (G) hTERT-BJ1 cells inoculated with culture supernatant collected from mock-infected NSPC/iPSCs (upper panel) or Towne HCMV-infected NSPC/iPSCs (lower panel) at 8 dpi were subjected to immunofluorescence test with anti-IE1/IE2 antibody (green). Nuclei were stained with DAPI. Representative results from two independent experiments are shown. 
also at the protein level by immunoblot analysis on 1, 2, 5, and 7 dpi (Figure 2F). In accordance with the results with RT-PCR, expression of Pax6 and Nestin was confirmed, and that of Nestin was found markedly decreased $7 \mathrm{dpi}$. Another NSPC marker Musashi-1 was also detected. To examine whether HCMV-infected NSPC/iPSCs produce infectious virions, culture supernatants collected from Towne HCMV-infected NSPC/iPSCs were inoculated to hTERT-BJ1 cells. Inoculated cells expressed IE1/IE2 indicating that infectious virions were produced from HCMV-infected NSPC/iPSCs (Figure 2G). The supernatant contained $30 \mathrm{PFU} / \mathrm{mL}$ of $\mathrm{HCMV}$ at $4,6,8 \mathrm{dpi}$, while no plaque forming virus was detected at $2 \mathrm{dpi}$.

\section{HCMV infection induces apoptosis in iPSC-derived NSPCs}

To examine whether HCMV infection in NSPC/iPSCs induces apoptotic responses, we performed the TUNEL assay combined with IFA using an antibody specific to HCMV gB. As shown in Figure 3A, NSPC/iPSCs expressing $\mathrm{gB}$ was positive for TUNEL staining and those without gB expression was consistently negative. We also performed IFA to analyze the activation status of caspases using antibodies specific to the activated forms of caspase-3 and caspase-9. The results show that the activated forms of caspase- 3 and caspase- 9 were specifically detected in more than $80 \%$ of HCMV-infected NSPC/iPSCs expressing IE1/IE2 proteins (Figure 3B and $3 C$ ), but not in mock-infected NSPC/iPSCs (Figure 3E). To see whether mitochondrial dysfunction is involved in the activation of caspase 9, intracellular distribution of cytochrome c was analyzed in HCMV-infected cells by IFA. As shown in Figure $3 \mathrm{D}$ and $3 \mathrm{E}$, strong signals of cytochrome $\mathrm{c}$ were detected in the cytosol of cells expressing IE1/IE2 proteins, while only faint signals of cytochrome c were detected in cells not expressing IE1/ IE2 proteins or in mock-infected cells. These results indicate that HCMV infection of NSPC/iPSCs activated apoptotic responses involving release of mitochondrial cytochrome $\mathrm{c}$ and serial activation of caspases.

\section{Unfolded protein response in HCMV-infected NSPC/iPSCs}

The unfolded protein response (UPR), induced by the accumulation of improperly folded proteins within the ER lumen (ER stress), is associated with multiple cellular responses such as neurodegeneration and apoptosis. ER stress sensor molecules, such as PKR-like eukaryotic initiation factor 2a kinase (PERK) and inositol-requiring enzyme 1 (IRE1), are activated on UPR and engage downstream signaling pathways. To examine whether the caspase- 9 activation in HCMV-infected NSPC/iPSCs (Figure 3C) is associated with UPR, we analyzed phosphorylation status of IRE1 $\alpha$ and its downstream target c-Jun NH2-terminal kinase (JNK) in immunofluorescence assays. Both IRE1 $\alpha$ and JNK were specifically phosphorylated in
HCMV-infected NSPC/iPSCs (Figure 4A and 4B), but not in mock-infected NSPC/iPSCs (Figure 4C). In concordance with the previous reports that activated IRE1 $\alpha$ catalyzes the non-conventional splicing of the mRNA encoding X-box binding protein 1 (XBP1) [20,21], the spliced XBP1 mRNA increased gradually after HCMV infection in NSPC/iPSCs (Figure 4D). We also analyzed phosphorylation status of another sensor molecule PERK, an ER-associated serine/ threonine protein kinase, and its downstream target the alpha subunit of eukaryotic initiation factor 2 (eIF2 $\alpha$ ). Phosphorylated forms of PERK and eIF2 $\alpha$ were specifically detected in HCMV-infected NSPC/iPSCs (Figure 4E and 4F), but not in mock-infected NSPC/iPSCs (Figure 4G). The transcription factor activating transcription factor 4 (ATF4), that is preferentially translated on activation of PERK, induces the expression of C/EBP-homologous protein (CHOP/GADD153), a transcription factor with proapoptotic functions [22]. In accordance with these previous findings, the mRNA level of $\mathrm{CHOP}$ increased gradually after HCMV infection in NSPC/iPSCs (Figure 4H). These results suggest that UPR is involved in the activation of caspase cascade leading to apoptosis in HCMV-infected NSPC/iPSCs.

\section{Discussion}

Important findings in this study are as follows: i) NSPC/ iPSCs derived from MRC-iPS-25 cells were susceptible to HCMV infection and allow the expression of viral gene products of both early and late functions and production of infectious virions. In contrast, MRC-iPS-25 cells before induction of differentiation was either resistant to HCMV or did not support the expression of HCMV immediateearly genes; ii) the HCMV-infected NSPCs undergo apoptosis; and iii) the mechanism of the apoptosis included cytochrome c release from mitochondria to cytosol and activation of UPR-related signaling pathways.

Neuropathogenesis of HCMV infection has been studied mainly with neural cells isolated from human brain. These studies demonstrated that HCMV can infect human neural precursor cells (NPCs) isolated from fetal brains and interfere with their differentiation. Luo et al. [23] showed that HCMV infection in primary NPCs reduced the expression of Nestin, suggesting that HCMV affects the differentiation potential of NPCs. Similar results were also obtained from experiments with mouse NSCs infected with MCMV [10,13,24]. Those previous findings obtained from experiments with primary cultures of brain-derived neural cells were thus mostly reproduced in our experiments using NSPC/iPSCs. In addition, similar to the results of Odeberg et al. [8] that used NPCs derived from human brain, we also demonstrated that HCMV infection induced apoptosis in NSPC/iPSCs obtained from iPSCs. It is thus supposed that neural cells differentiated from iPSCs are a useful 

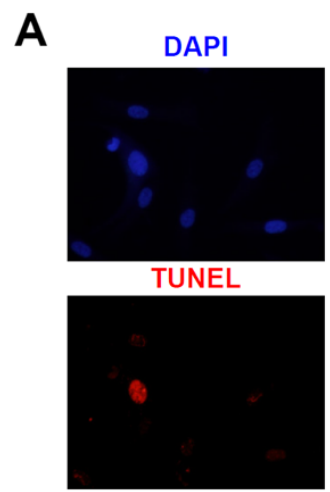

C

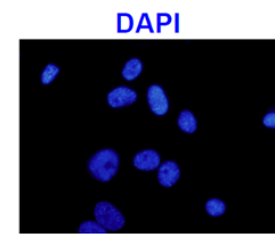

cleaved caspase 9

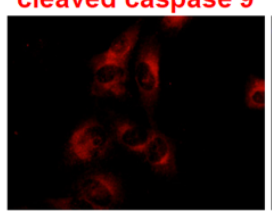

E

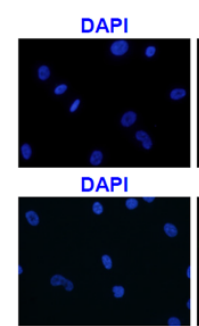

DAPI

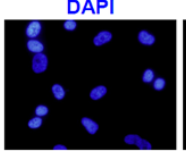

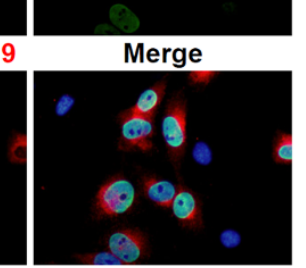

gB

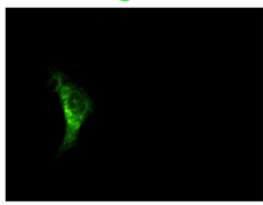

Merge

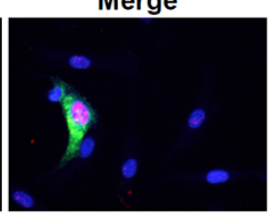

IE1/IE2
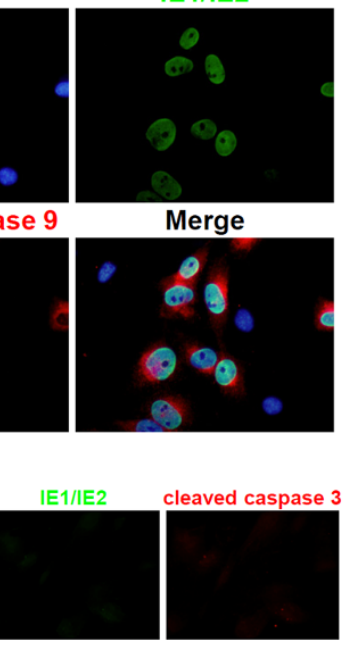

IE1/IE2

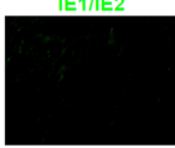

IE1/IE2

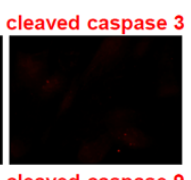

cleaved caspase 9

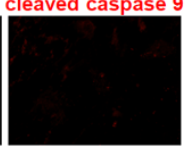

cytochrome c
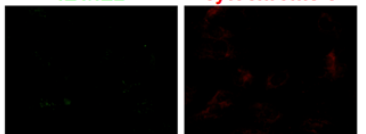

B
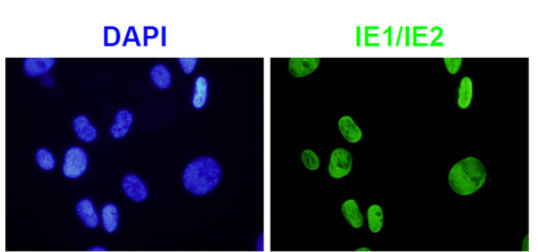

cleaved caspase 3
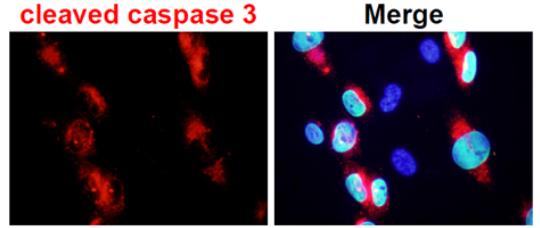

D

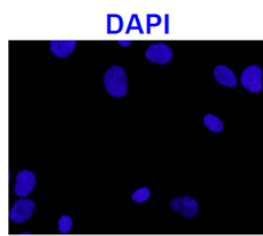

cytochrome c
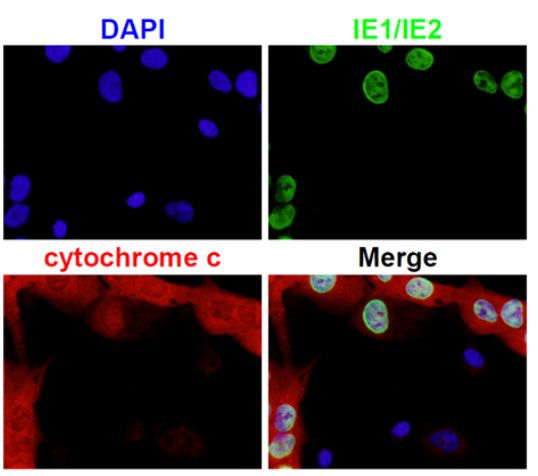

Figure 3 HCMV-induced apoptosis of NSPC/iPSCs. (A) Towne-infected NSPC/iPSCs at 6 dpi were subjected to TUNEL assay followed by immunofluorescence test with an anti-gB antibody. TUNEL-positive nuclei were stained in red. The anti-gB antibody was detected with Alexa Fluor 488-conjugated goat anti-mouse lgG antibody (green). Nuclei were stained with DAPI. (B-D) Towne-infected NSPC/iPSCs at 3 dpi were subjected to immunofluorescence test with anti-IE1/IE2 antibody in combination with anti-cleaved caspase 3 (B), anti-cleaved caspase 9 (C), or anti-cytochrome C (D) antibody. Alexa Fluor 488-conjugated goat anti-mouse lgG (green) or Alexa Fluor 594-conjugated goat anti-rabbit lgG antibody (red) was used as a secondary antibody. Nuclei were stained with DAPI. (E) Mock-infected NSPC/iPSCs were subjected to immunofluorescence test with anti-IE1/IE2 antibody in combination with anti-cleaved caspase 3 (upper panel), anti-cleaved caspase 9 (middle panel), or anti-cytochrome c (lower panel) antibody. Nuclei were stained with DAPI. Representative results from two independent experiments are shown.

model to investigate neural pathogenesis of HCMV. In the human brain, NSCs are predominantly found in the subventricular region where CMV infections preferentially occur $[25,26]$. Analysis on the effects of HCMV infection on NSPCs can be therefore particularly relevant.

In the regulation of cellular apoptotic responses, mitochondrial dysfunction and ER stress are involved in the activation of the initiator caspase caspase- 9 that functions as a trigger of cascade protease reactions leading to cell death. The finding of cytochrome $\mathrm{c}$ release from mitochondria to cytoplasm in HCMV-infected NSPC/iPSCs indicates that mitochondrial dysfunction is involved in the activation of caspase- 9 in these cells. In addition, the demonstration of phosphorylated forms of proteins involved in UPR, including PERK, JNK, IRE1 $\alpha$, eIF2 $\alpha$, as well as that of unconventional splicing of 


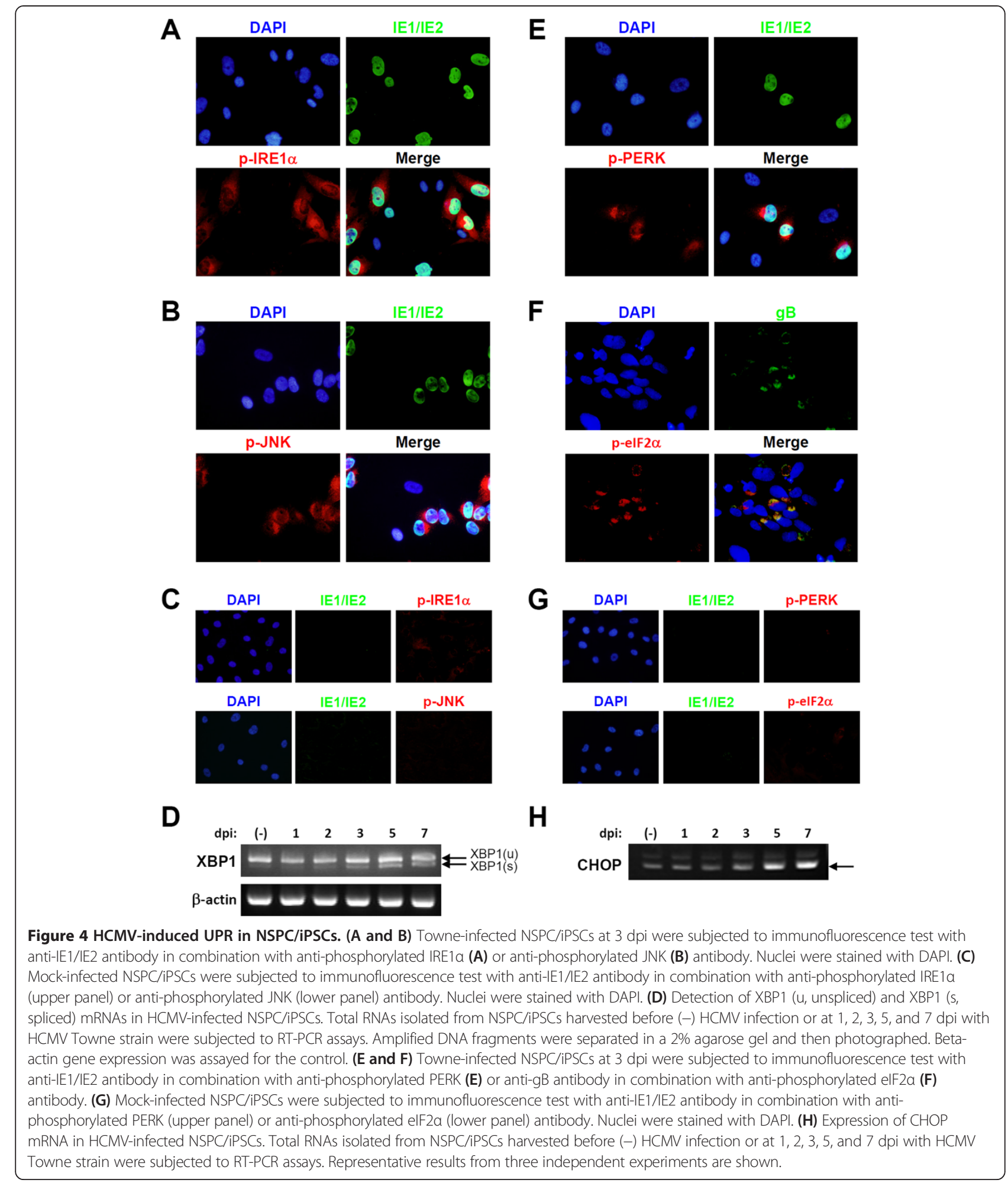

XBP1 mRNA and up-regulation of CHOP, indicate that ER stress also plays a role in HCMV-induced apoptosis of NSPC/iPSCs. These results are in accordance with the work reported by Isler et al. [27] who demonstrated that HCMV-induced UPR in human foreskin fibroblasts.
HCMV is known to encode anti-apoptotic proteins such as viral inhibitor of caspase-8-induced apoptosis (vICA) encoded by UL36 [28], and pUL38 which protects against ER stress-induced cell death by modulating the UPR pathway [29]. Our RT-PCR analysis demonstrated 
that such viral anti-apoptotic genes were expressed at transcription level in NSPC/iPSCs following HCMV infection (Figure 2B). Although these viral anti-apoptotic proteins did not block apoptosis of NSPC/iPSCs, they might have contributed for efficient viral replication by delaying apoptosis.

iPSCs are expected to be an innovative tool for not only regenerative medicine but also for the elucidation of pathogenesis of various diseases. Recent studies have shown that human iPSCs can be used also for modeling viral infection. Hepatocyte-like cells derived from human iPSCs were shown to be susceptible to hepatitis virus $C$ infection and supported its replication [30,31]. Sensory neurons derived from human iPSCs were reported to be susceptible to infection with both varicella-zoster virus and herpes simplex virus [32]. While the present work was in progress, D'Aiuto and others reported on the preparation of an iPSC-derived model of HCMV infection in neural precursor cells [33]. Whereas our data described in the present study is largely consistent with their results, we further analyzed the mechanisms of apoptosis induction and elucidated the involvement of mitochondrial dysfunction and ER stress.

In conclusion, human NSPCs derived from iPSCs can be a useful model to study HCMV neuropathogenesis associated with congenital HCMV infection. They can be particularly valuable in analyzing the mechanisms of HCMV-induced apoptosis in neural cells.

\begin{abstract}
Abbreviations
HCMV: Human cytomegalovirus; iPSC: Induced pluripotent stem cell; ESC: Embryonic stem cell; NSPC: Neural stem/progenitor cell;

TUNEL: Terminal deoxynucleotidyl transferase-mediated dUTP nick-end labeling; UPR: Unfolded protein response; ER: Endoplasmic reticulum; PERK: PKR-like eukaryotic initiation factor 2a kinase; JNK: c-Jun NH2-terminal kinase; IRE1: Inositol-requiring enzyme 1; elF2a: Alpha subunit of eukaryotic initiation factor 2; CHOP: C/EBP-homologous protein; XBP1: X-box binding protein 1; IFA: Indirect immunofluorescence assay; Dpi: Days post-infection; MAP2: Microtubule-associated protein 2; GFAP: Glial fibrillary acidic protein; OSP: Oligodendrocyte-specific protein; ATF4: Activating transcription factor 4; MOI: Multiplicity of infection.
\end{abstract}

\section{Competing interests}

The authors declare that they have no competing interests.

\section{Authors' contributions}

$\mathrm{HN}, \mathrm{HL}, \mathrm{KM}$, and $\mathrm{HA}$ performed the experimental studies, and $\mathrm{KI}$ helped to analyze the data. $\mathrm{KM}, \mathrm{MT}, \mathrm{HA}, \mathrm{YM}, \mathrm{HO}, \mathrm{NK}$, and $\mathrm{AU}$ participated in the characterization of iPSCs and their derivatives. HN, HL, and SF wrote the manuscript. NI revised the manuscript. All authors read and approved the final manuscript.

\section{Acknowledgments}

We especially thank M. Katata for excellent technical assistance. This work was partly supported by Grants-in-Aid for Scientific Research from the Ministry of Education, Culture, Sports, Science and Technology of Japan (24591616), the Ministry of Health, Labour and Welfare of Japan (H23-JisedaiIppan-001), and the Grants of National Center for Child Health and Development (22A-9 and 24-17)

\section{Author details}

'Department of Infectious Diseases, National Research Institute for Child Health and Development, 2-10-1 Okura, Setagaya-ku, Tokyo 157-8535, Japan. ${ }^{2}$ Department of Reproductive Biology, Center for Regenerative Medicine, National Research Institute for Child Health and Development, 2-10-1 Okura, Setagaya-ku, Tokyo 157-8535, Japan. ${ }^{3}$ Department of Pediatric Hematology and Oncology Research, National Research Institute for Child Health and Development, 2-10-1 Okura, Setagaya-ku, Tokyo 157-8535, Japan.

${ }^{4}$ Department of Virology I, National Institute of Infectious Diseases, 1-23-1 Toyama, Shinjuku-ku, Tokyo 162-8640, Japan.

Received: 17 April 2013 Accepted: 15 October 2013

Published: 21 October 2013

\section{References}

1. Cheeran MC, Lokensgard JR, Schleiss MR: Neuropathogenesis of congenital cytomegalovirus infection: disease mechanisms and prospects for intervention. Clin Microbiol Rev 2009, 22(1):99-126. Table of Contents.

2. Revello MG, Gerna G: Diagnosis and management of human cytomegalovirus infection in the mother, fetus, and newborn infant. Clin Microbiol Rev 2002, 15(4):680-715

3. Koyano S, Inoue N, Oka A, Moriuchi H, Asano K, Ito Y, Yamada H, Yoshikawa $T$, Suzutani T: Screening for congenital cytomegalovirus infection using newborn urine samples collected on filter paper: feasibility and outcomes from a multicentre study. BMJ Open 2011, 1(1):e000118.

4. Cinatl J Jr, Vogel JU, Cinatl J, Weber B, Rabenau H, Novak M, Kornhuber B, Doerr HW: Long-term productive human cytomegalovirus infection of a human neuroblastoma cell line. Int J Cancer 1996, 65(1):90-96.

5. Cinatl J Jr, Cinatl J, Vogel JU, Kotchetkov R, Driever PH, Kabickova H, Kornhuber B, Schwabe D, Doerr HW: Persistent human cytomegalovirus infection induces drug resistance and alteration of programmed cell death in human neuroblastoma cells. Cancer Res 1998, 58(2):367-372.

6. Luo $\mathrm{MH}$, Fortunato EA: Long-term infection and shedding of human cytomegalovirus in T98G glioblastoma cells. J Virol 2007, 81(19):10424-10436.

7. Ogura T, Tanaka J, Kamiya S, Sato H, Ogura H, Hatano M: Human cytomegalovirus persistent infection in a human central nervous system cell line: production of a variant virus with different growth characteristics. J Gen Virol 1986, 67(Pt 12):2605-2616.

8. Odeberg J, Wolmer N, Falci S, Westgren M, Seiger A, Soderberg-Naucler C: Human cytomegalovirus inhibits neuronal differentiation and induces apoptosis in human neural precursor cells. J Virol 2006, 80(18):8929-8939.

9. Odeberg J, Wolmer N, Falci S, Westgren M, Sundtrom E, Seiger A, Soderberg-Naucler C: Late human cytomegalovirus (HCMV) proteins inhibit differentiation of human neural precursor cells into astrocytes. J Neurosci Res 2007, 85(3):583-593.

10. Cheeran MC, Jiang Z, Hu S, Ni HT, Palmquist JM, Lokensgard JR: Cytomegalovirus infection and interferon-gamma modulate major histocompatibility complex class I expression on neural stem cells. J Neurovirol 2008, 14(5):437-447.

11. Matsukage $S$, Kosugi I, Kawasaski H, Miura K, Kitani H, Tsutsui Y: Mouse embryonic stem cells are not susceptible to cytomegalovirus but acquire susceptibility during differentiation. Birth Defects Res A Clin Mol Teratol 2006, 76(2):115-125

12. Li RY, Tsutsui Y: Growth retardation and microcephaly induced in mice by placental infection with murine cytomegalovirus. Teratology 2000, 62(2):79-85.

13. Kosugi I, Shinmura Y, Kawasaki H, Arai Y, Li RY, Baba S, Tsutsui Y: Cytomegalovirus infection of the central nervous system stem cells from mouse embryo: a model for developmental brain disorders induced by cytomegalovirus. Lab Invest 2000, 80(9):1373-1383.

14. Nishino K, Toyoda M, Yamazaki-Inoue M, Fukawatase Y, Chikazawa E, Sakaguchi H, Akutsu H, Umezawa A: DNA methylation dynamics in human induced pluripotent stem cells over time. PLoS Genet 2011, 7(5):e1002085.

15. Makino H, Toyoda M, Matsumoto K, Saito H, Nishino K, Fukawatase $Y$, Machida M, Akutsu H, Uyama T, Miyagawa $Y$, et al: Mesenchymal to embryonic incomplete transition of human cells by chimeric OCT4/3 (POU5F1) with physiological co-activator EWS. Exp Cell Res 2009, 315(16):2727-2740.

16. Chambers SM, Fasano CA, Papapetrou EP, Tomishima M, Sadelain M, Studer $L$ : Highly efficient neural conversion of human ES and iPS cells by dual inhibition of SMAD signaling. Nat Biotechnol 2009, 27(3):275-280. 
17. Nakamura H, Lu M, Gwack Y, Souvlis J, Zeichner SL, Jung JU: Global changes in Kaposi's sarcoma-associated virus gene expression patterns following expression of a tetracycline-inducible Rta transactivator. J Virol 2003, 77(7):4205-4220.

18. White EA, Clark CL, Sanchez V, Spector DH: Small internal deletions in the human cytomegalovirus IE2 gene result in nonviable recombinant viruses with differential defects in viral gene expression. J Virol 2004, 78(4):1817-1830

19. Pevny LH, Sockanathan S, Placzek M, Lovell-Badge R: A role for SOX1 in neural determination. Development 1998, 125(10):1967-1978.

20. Calfon M, Zeng H, Urano F, Till JH, Hubbard SR, Harding HP, Clark SG, Ron D: IRE1 couples endoplasmic reticulum load to secretory capacity by processing the XBP-1 mRNA. Nature 2002, 415(6867):92-96.

21. Sidrauski $C$, Walter $P$ : The transmembrane kinase Ire $1 p$ is a site-specific endonuclease that initiates mRNA splicing in the unfolded protein response. Cell 1997, 90(6):1031-1039.

22. Scheuner D, Song B, McEwen E, Liu C, Laybutt R, Gillespie P, Saunders T, Bonner-Weir S, Kaufman RJ: Translational control is required for the unfolded protein response and in vivo glucose homeostasis. Mol Cell 2001, 7(6):1165-1176.

23. Luo MH, Hannemann H, Kulkarni AS, Schwartz PH, O'Dowd JM, Fortunato EA: Human cytomegalovirus infection causes premature and abnormal differentiation of human neural progenitor cells. J Virol 2010, 84(7):3528-3541.

24. Mutnal MB, Cheeran MC, Hu S, Lokensgard JR: Murine cytomegalovirus infection of neural stem cells alters neurogenesis in the developing brain. PLoS One 2011, 6(1):e16211.

25. Grassi MP, Clerici F, Perin C, D'Arminio Monforte A, Vago L, Borella M, Boldorini R, Mangoni A: Microglial nodular encephalitis and ventriculoencephalitis due to cytomegalovirus infection in patients with AIDS: two distinct clinical patterns. Clin Infect Dis 1998, 27(3):504-508.

26. Perlman JM, Argyle C: Lethal cytomegalovirus infection in preterm infants: clinical, radiological, and neuropathological findings. Ann Neurol 1992, 31(1):64-68.

27. Isler JA, Skalet AH, Alwine JC: Human cytomegalovirus infection activates and regulates the unfolded protein response. J Virol 2005, 79(11):6890-6899.

28. Skaletskaya A, Bartle LM, Chittenden T, McCormick AL, Mocarski ES, Goldmacher VS: A cytomegalovirus-encoded inhibitor of apoptosis that suppresses caspase-8 activation. Proc Natl Acad Sci U S A 2001, 98(14):7829-7834.

29. Terhune S, Torigoi E, Moorman N, Silva M, Qian Z, Shenk T, Yu D: Human cytomegalovirus UL38 protein blocks apoptosis. J Virol 2007, 81(7):3109-3123.

30. Schwartz RE, Trehan K, Andrus L, Sheahan TP, Ploss A, Duncan SA, Rice CM Bhatia SN: Modeling hepatitis $C$ virus infection using human induced pluripotent stem cells. Proc Natl Acad Sci U S A 2012, 109(7):2544-2548.

31. Wu X, Robotham JM, Lee E, Dalton S, Kneteman NM, Gilbert DM, Tang H: Productive hepatitis $\mathrm{C}$ virus infection of stem cell-derived hepatocytes reveals a critical transition to viral permissiveness during differentiation. PLoS Pathog 2012, 8(4):e1002617.

32. Lee KS, Zhou W, Scott-McKean JJ, Emmerling KL, Cai GY, Krah DL, Costa AC, Freed CR, Levin MJ: Human sensory neurons derived from induced pluripotent stem cells support varicella-zoster virus infection. PLoS One 2012, 7(12)::53010

33. D'Aiuto L, Di Maio R, Heath B, Raimondi G, Milosevic J, Watson AM, Bamne $M$, Parks WT, Yang L, Lin B, et al: Human induced pluripotent stem cellderived models to investigate human cytomegalovirus infection in neural cells. PLoS One 2012, 7(11):e49700.

doi:10.1186/2042-4280-4-2

Cite this article as: Nakamura et al:: Human cytomegalovirus induces apoptosis in neural stem/progenitor cells derived from induced pluripotent stem cells by generating mitochondrial dysfunction and endoplasmic reticulum stress. Herpesviridae 2013 4:2.

\section{Submit your next manuscript to BioMed Central and take full advantage of:}

- Convenient online submission

- Thorough peer review

- No space constraints or color figure charges

- Immediate publication on acceptance

- Inclusion in PubMed, CAS, Scopus and Google Scholar

- Research which is freely available for redistribution

Submit your manuscript at www.biomedcentral.com/submit
Biomed Central 